\title{
Trends in Pathogen Occurrence and Antimicrobial Resistance of Urinary Isolates in a Tertiary Medical Center over Ten Years: 2004 2013
}

\author{
Seung Bok Hong ${ }^{1}$, Jong Hwa Yum ${ }^{2}$, Yong Dae Kim ${ }^{3}$ and Kyeong Seob Shin ${ }^{4, \uparrow}$ \\ ${ }^{I}$ Department of Clinical Laboratory Science, Chungbuk Health \& Science University, Cheong-ju 363-794, Korea \\ ${ }^{2}$ Department of Clinical Laboratory Science, Dong-eui University, Busan 614-714, Korea \\ ${ }^{3}$ Department of Preventive Medicine, Chungbuk National University College of Medicine, Cheongju 362-763, Korea \\ ${ }^{4}$ Department of Laboratory Medicine, Chungbuk National University College of Medicine, Cheongju 362-763, Korea
}

To provide guidelines for the empirical treatment of urinary tract infections, we observed annual changes in the occurrence frequency and antimicrobial susceptibility of urinary isolates in a university hospital in the Chungbuk province, South Korea, over a period of 10 years (2004 2013). Escherichia coli (38.2\%), Enterococcus faecalis (11.7\%), Klebsiella pneumoniae (7.3\%), Pseudomonas aeruginosa (4.3\%), E. faecium (4.3\%), and Staphylococcus aureus (4.1\%) were commonly isolated urinary pathogens. The prevalence of E. coli, E. faecium and Streptococcus agalactiae were significantly higher in females $(P<0.001)$, whereas $E$. faecalis, $P$. aeruginosa and $S$. aureus were significantly more common in male patients $(P<0.001)$. E. coli mostly frequently showed resistance to ampicillin $(67.94 \%)$, followed by trimethoprim/sulfamethoxazole (36.06\%) and ciprofloxacin (26.84\%). Over the studied time period, resistance rates of E. coli to ciprofloxacin significantly increased (20.44\% to $33.55 \%)$. Moreover, extended-spectrum $\beta$-lactamase (ESBL) producing isolates also significantly increased in $E$. coli ( $4.2 \%$ to $18.3 \%$ ) and $K$. pneumoniae ( $9.6 \%$ to $26.9 \%)$. In addition, the proportion of vancomycin-resistant Enterococcus facium (VRE) also increased (15.7\% to 25.0\%). In conclusion, over the last 10 years, the proportions of ciprofloxacin resistant $E$. coli and multidrug-resistant bacteria, such as ESBL and VRE have significantly increased. This trend must be strictly controlled and demonstrates the need for more updated guidelines for the treatment of urinary tract infections.

Key Words: Urinary tract infection, Occurrence, Antimicrobial susceptibility, Trend

\section{INTRODUCTION}

Urinary tract infections (UTIs) are the most common community- and hospital-acquired infections. Uncomplicated UTIs typically affect young women who are immunocompetent and have anatomically normal physiology (Warren

*Received: May 7, 2015 / Revised: May 29, 2015

Accepted: June 6, 2015

${ }^{\dagger}$ Corresponding author: Kyeong Seob Shin. Department of Laboratory Medicine, Chungbuk National University College of Medicine, Cheongju 362-763, Korea.

Tel: +82-43-269-6240, Fax: +82-43-271-5243

e-mail: ksshin@chungbuk.ac.kr

(C)The Korean Society for Biomedical Laboratory Sciences. All rights reserved.
2001). Complicated UTIs often affect patients with underlying functional and metabolic or anatomical defects of the urinary tract. In addition, most nosocomial UTIs are related to catheterization (Ronald 2003). The etiologic bacteria of UTIs are predictable, with Escherichia coli being the principle pathogen (Hooton et al., 1999; Warren et al., 1999). Klebsiella pneumoniae, Pseudomonas aeruginosa and Enterococcus spp. are also commonly isolated (Warren et al., 1999; Turnidge et al., 2002; Shin et al., 2005).

E. coli and other uropathogens are becoming increasingly resistant to commonly used antimicrobial agents, reducing the effectiveness of some standard regimens (Thomson et al., 1994; Friedrich et al., 1999; Gupta et al., 1999; Hooton 
et al., 1999; Jones et al., 1999; Warren et al., 1999). Meanwhile, epidemiologic and resistance patterns of the pathogens in UTIs show inter-regional variability, and the susceptibility patterns are continually changing depending on different regional antibiotic treatment regimens (Karlowsky et al., 2002; den Heijer et al., 2010). In most cases of UTI, empirical antibiotic therapy is initiated before the laboratory results of urine cultures are received. Such therapy should be tailored to the surveillance data on the epidemiology and resistance patterns of common uropathogens to reduce treatment failures and the emergence of bacterial resistant strains (Gupta 2002). In the present study, we examined changes in the pattern of the causative organisms of UTIs isolated from patients in a university hospital.

\section{MATERIALS AND METHODS}

\section{Retrospective clinical study}

This was a retrospective study based on laboratory records over 10 years from 2004 to 2013 at the Chungbuk National University Hospital, Cheongju, Republic of Korea, which is a tertiary teaching hospital with 600 beds and has 173,000 admissions a year. Only an isolate of pathogens that were repeatedly isolated from patients were included. All patients with a suspected UTI were examined. Significant bacteriuria was defined as a urine specimen containing more than $10^{5}$ $\mathrm{CFU} / \mathrm{mL}$

\section{Urine culture}

The identification of organisms was performed using the VITEK II automatic analyzer (bioMérieux Inc., Hazelwood, $\mathrm{MO}, \mathrm{USA}$ ) and routine biochemical tests.

\section{Antimicrobial susceptibility testing}

Antimicrobial susceptibility testing (AST) was done with the VITEK II system or the Kirby Bauer disc diffusion method using Muller Hinton agar. Quality-control tests of AST ware performed using standard strains of Escherichia coli ATCC 25922, Pseudomonas aeruginosa ATCC 27953, Staphylococcus aureus ATCC 25923 and Enterococcus faecalis ATCC 29212 as per the recommendations of the Clinical Laboratory Standard Institute (CLSI, 2012). The antimicrobial susceptibility against each antimicrobial agent was interpreted as CLSI guideline. When vancomycin resistance in Gram-positive cocci or imipenem resistance in Gram-negative bacilli was observed in the VITEK II system or the disc diffusion method, the results were further confirmed using the E-test (bioMérieux, Inc., Durham, NC, USA).

\section{Statistical analysis}

The data were collected from the laboratory information system of the hospital and were analyzed using Microsoft Excel 7.0 software. Differences in proportions of UTI isolates by gender and trend in change of antimicrobial susceptibilities were compared using a chi-square test with SPSS 19.0. Statistical significance was reached at $P<0.05$.

\section{RESULTS}

\section{Distribution of urine culture positive isolates}

Of 106,269 cultured urine specimens, 19,075 (17.9\%) were positive. Among the positive isolates, 93.4\% were bacteria, and $6.6 \%$ were fungi. The overall female to male ratio was 1.19:1. A significant gender-dependent difference, with a two-fold male dominance was observed for $E$. faecalis, $P$. aeruginosa and $S$. aureus, and a two-fold female dominance was noted for E. coli (Table 1).

Among the pathogens, the most common 10 isolates were: $E$. coli (7,283 cases, 38.2\%), E. faecalis (2,231 cases, 11.7\%), Klebsiella pneumoniae (1,398 cases, 7.3\%), P. aeruginosa (828 cases, 4.3\%), E. faecium (814 cases, 4.3\%), S. aureus (778 cases, 4.1\%), Candida species not albicans (650 cases, 3.4\%), Candida albicans (615 cases, 3.2\%), Morganella morganii (487 cases, 2.6\%), and Enterobacter aerogenes (358 cases, $1.9 \%$ ) (Table 1$)$.

\section{Antimicrobial resistance patterns of positive isolates}

Antimicrobial resistance trends are presented in Table 2. E. coli was frequently resistant to ampicillin (67.94\%). The resistance rate against ciprofloxacin and trimethoprim/ sulfamethoxazole (SXT) increased to more than 30\% (33.55\%, $38.9 \%$, respectively). In addition, the resistance rate of $E$. coli to cefazolin and cefotaxime was increased from $11.5 \%$ 
and $3.72 \%$ in $2004 \sim 5$ to $25.63 \%$ and $21.32 \%$, respectively in 2012 3 (Table 2, Fig. 1). The resistance rate of K. pneumoniae for cefotaxime increased from $11.34 \%$ in $2004 \sim 5$ to $29.05 \%$ in $2012 \sim 3$ (Table 2). A constant annual increase in ESBL producing E. coli and K. pneumoniae was observed over the 10-year time period (Fig. 2). P. aeruginosa was frequently resistant to ciprofloxacin $(37.32 \%)$, imipenem (24.64.9\%), and ceftazidime $(22.71 \%)$. The resistance rate of imipenem was rather decreased from $33.33 \%$ in 2004 2005 to $24.38 \%$ in $2012 \sim 3$ (Table 2).

Table 1. Frequency and distribution of isolates causing urinary tract infection over a period of 10 years (2004 2013)

\begin{tabular}{|c|c|c|c|c|}
\hline \multirow{2}{*}{ Microorganism } & \multicolumn{3}{|c|}{ Isolates, N(\%) } & \multirow{2}{*}{$P$-value } \\
\hline & Male $(45.61 \%)$ & Female $(54.39 \%)$ & Total & \\
\hline E. coli & $2,160(24.83)$ & $5,123(49.38)$ & $7,283(38.18)$ & $<0.001$ \\
\hline E. faecalis & $1,338(15.38)$ & $893(8.61)$ & $2,231(11.70)$ & $<0.001$ \\
\hline K. pneumoniae & $645(7.41)$ & $753(7.26)$ & $1,398(7.33)$ & NS \\
\hline P. aeruginosa & $533(6.13)$ & $295(2.84)$ & $828(4.34)$ & $<0.001$ \\
\hline E. faecium & $270(3.10)$ & $544(5.24)$ & $814(4.27)$ & $<0.001$ \\
\hline S. aureus & $494(5.68)$ & $284(2.74)$ & $778(4.08)$ & $<0.001$ \\
\hline C. spp. NA & $312(3.59)$ & $338(3.26)$ & $650(3.41)$ & NS \\
\hline C. albicans & $313(3.60)$ & $302(2.91)$ & $615(3.22)$ & 0.007 \\
\hline M. morganii & $361(4.15)$ & $126(1.21)$ & $487(2.55)$ & $<0.001$ \\
\hline E. aerogenes & $199(2.29)$ & $159(1.53)$ & $358(1.88)$ & $<0.001$ \\
\hline S. agalactiae & $107(1.23)$ & $230(2.22)$ & $337(1.77)$ & $<0.001$ \\
\hline A. baumanii & $232(2.67)$ & $96(0.93)$ & $328(1.72)$ & $<0.001$ \\
\hline P. mirabilis & $180(2.08)$ & $145(1.40)$ & $326(1.71)$ & $<0.001$ \\
\hline C. freundii & $189(2.17)$ & $131(1.26)$ & $320(1.68)$ & $<0.001$ \\
\hline E. cloacae & $173(1.99)$ & $135(1.30)$ & $308(1.61)$ & $<0.001$ \\
\hline K. oxytoca & $204(2.34)$ & $84(0.81)$ & $288(1.51)$ & $<0.001$ \\
\hline S. marcescens & $137(1.57)$ & $34(0.33)$ & $171(0.90)$ & $<0.001$ \\
\hline Others & 852 (9.79) & $703(6.78)$ & $1,555(8.15)$ & $<0.001$ \\
\hline Total & $8,700(100)$ & $10,375(100)$ & $19,075(100)$ & \\
\hline
\end{tabular}

Abbreviations: N, number; NS, not significant: NA, not albicans

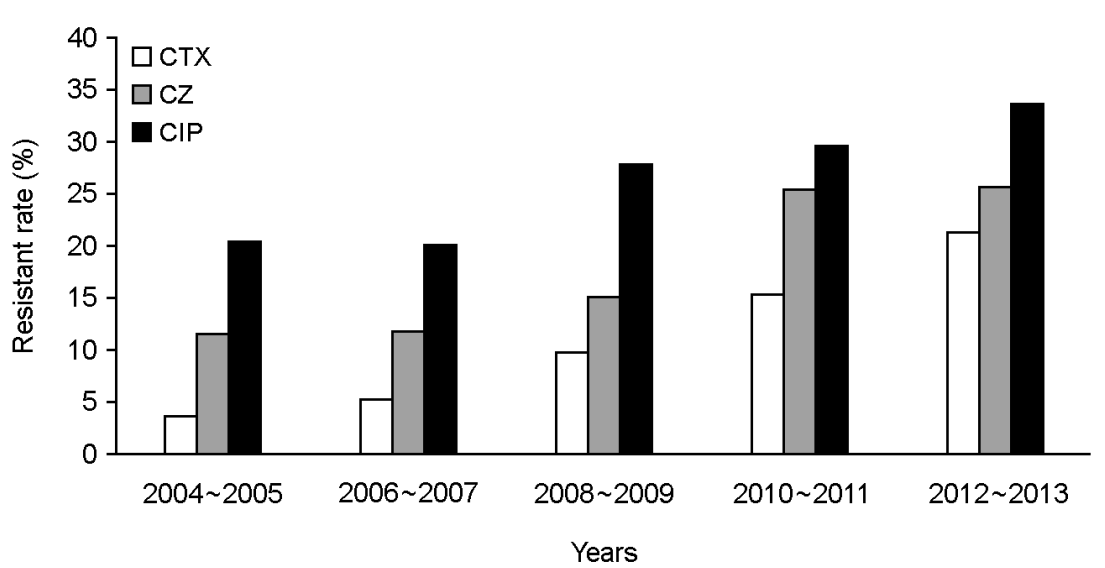

$-86-$
Fig. 1. Trends of resistance rate $(\%)$ against cefotaxime (CTX), cefazolin (CZ) and ciprofloxacin (CIP) in E. coli isolated from urine culture with significant bacteriuria during 2004 2013 years. 
Table 2. Trends of antimicrobial resistance of common isolates causing urinary tract infection over 10 years

\begin{tabular}{|c|c|c|c|c|c|c|c|}
\hline \multirow{2}{*}{ Microorganisms } & \multicolumn{6}{|c|}{ Antimicrobial resistance rate $(\%)$} & \multirow[b]{2}{*}{$P$-value } \\
\hline & $2004 \sim 5$ & $2006 \sim 7$ & $2008 \sim 9$ & $2010 \sim 11$ & $2012 \sim 13$ & Mean of 10 years & \\
\hline \multicolumn{8}{|l|}{ SAU } \\
\hline CIP & 48.41 & 43.55 & 45.99 & 48.99 & 46.43 & 46.40 & 0.881 \\
\hline OXA & 68.25 & 73.12 & 74.33 & 80.58 & 69.29 & 73.26 & 0.156 \\
\hline PEN & 98.41 & 96.77 & 98.93 & 99.28 & 94.29 & 97.56 & 0.035 \\
\hline TET & 51.59 & 48.92 & 54.01 & 59.71 & 53.57 & 57.34 & 0.417 \\
\hline VAN & 0 & 0 & 0 & 0 & 0 & 0 & - \\
\hline \multicolumn{8}{|l|}{ EFA } \\
\hline AMP & 1.34 & 1.59 & 4.71 & NA & 0.33 & 2.31 & $<0.001$ \\
\hline VAN & 2.23 & 1.99 & 0.41 & 0.47 & 0.67 & 1.39 & 0.031 \\
\hline \multicolumn{8}{|l|}{ EFM } \\
\hline AMP & 95.10 & 88.24 & 96.34 & 98.56 & 97.16 & 95.58 & $<0.001$ \\
\hline VAN & 15.69 & 18.38 & 18.32 & 20.57 & 25.00 & 20.03 & 0.339 \\
\hline \multicolumn{8}{|l|}{ ECO } \\
\hline AMP & 69.56 & 64.22 & 66.96 & 69.99 & 69.07 & 67.94 & 0.004 \\
\hline AMK & 1.42 & 0.95 & 0.62 & 1.39 & 1.05 & 1.07 & 0.222 \\
\hline CIP & 20.44 & 20.07 & 27.87 & 29.61 & 33.55 & 26.84 & $<0.001$ \\
\hline CTX & 3.72 & 5.31 & 9.84 & 15.33 & 21.32 & 11.82 & $<0.001$ \\
\hline $\mathrm{CZ}$ & 11.56 & 11.90 & 15.21 & 25.45 & 25.63 & 18.56 & $<0.001$ \\
\hline SXT & 37.08 & 29.66 & 34.55 & 39.72 & 38.90 & 36.06 & $<0.001$ \\
\hline \multicolumn{8}{|l|}{ KPN } \\
\hline AMK & 12.55 & 7.37 & 5.95 & 10.31 & 4.39 & 8.01 & 0.003 \\
\hline CIP & 16.60 & 13.14 & 15.87 & 14.78 & 22.97 & 16.67 & 0.016 \\
\hline CTX & 11.34 & 9.29 & 19.44 & 26.80 & 29.05 & 19.31 & \\
\hline \multicolumn{8}{|l|}{ PAE } \\
\hline CAZ & 21.79 & 20.45 & 21.51 & 24.39 & 25.63 & 22.71 & 0.779 \\
\hline CIP & 44.87 & 38.07 & 34.88 & 35.37 & 33.75 & 37.32 & 0.247 \\
\hline IMP & 33.33 & 22.16 & 22.67 & 21.34 & 24.38 & 24.64 & 0.083 \\
\hline
\end{tabular}

Abbreviations: SAU, Staphylococcus aureus; CIP, ciprofloxacin; OXA, oxacillin; PEN, penicillin; TET, tetracycline; VAN, vancomycin; EFA, Enterococcus faecalis; AMP, ampicillin; EFM, Entercoccus faecium; ECO, Escherichia coli; AMK, amikacin; CTX, cefotaxime; CZ, cefazolin; KPN, Klebsiella pneumoniae; PAE, Pseudomonas aeruginosa; CAZ, ceftazidime; IMP, imipenem; ABA, Acinetobacter baumannii; NA, not available.

Vancomycin resistant S. aureus (VRSA) were not observed, although the average rate of MRSA over the 10 years did not significantly change (Table 2). The average resistance rates to ampicillin in E. faecalis and E. faecium were $2.31 \%$ and $95.58 \%$, respectively. The average vancomycin resistance rate of E. faecalis was $1.39 \%$, but that of $E$. faecium was $20.03 \%$. Among Gram positive cocci, a major change in the resistant rate was showed in VRE (from
$15.69 \%$ to $25.0 \%$ ) over the 10 -year study period (Table 2). In other microorganisms, only minor changes in resistant rates were noted.

\section{DISCUSSION}

Antibiotic resistance is now a major factor contributing to therapeutic failure in not only nosocomial complicated 


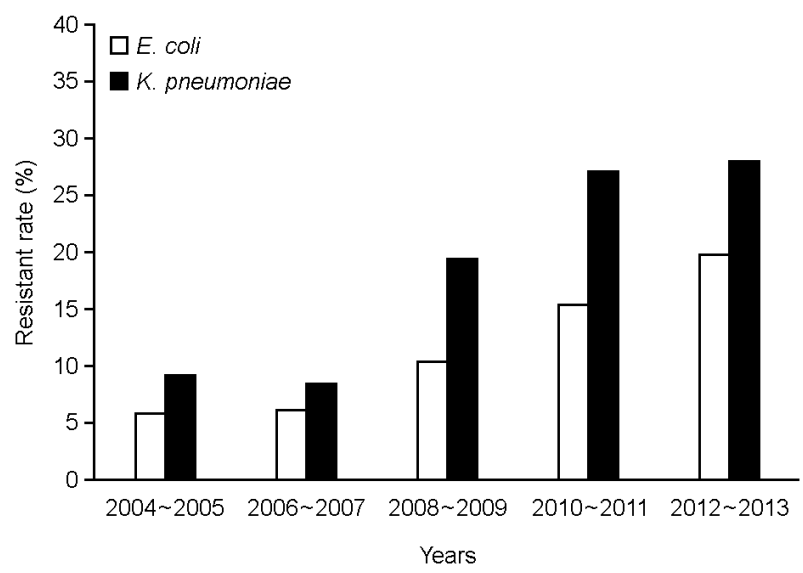

Fig. 2. Prevalence (\%) of ESBL producing E. coli and K. pneumoniae isolated from urine culture with significant bacteriuria during 2004 2013 years.

UTIs, but also uncomplicated community-acquired UTIs (Gupta 2002). Geographic variation in individual patient risk factors and an understanding of the resistance trends need to be incorporated into the management strategies for UTI.

The present study illustrates the UTI bacterial spectrum and antimicrobial susceptibility patterns in a university hospital over a period of 10 years. The average rate of positive urine culture was determined to be $17.9 \%$, and $E$. coli, E. faecalis, K. pneumoniae, P. aeruginosa, E. faecium, $S$. aureus were the most frequent causative pathogens. $E$. coli is the most common one associated with UTI, with a reported frequency of $38 \%$ to $85 \%$ depending on the study (Gupta et al., 1999; Farrel et al., 2003; Kahlmeter et al., 2003; Ti et al., 2003; Chazan et al., 2004; Shin et al., 2005). In addition, studies that have been limited to uncomplicated CA-UTIs or to female subjects only have reported a prevalence of E. coli of more than 50\% (Gupta et al., 1999; Farrel et al., 2003; Kahlmeter et al., 2003; Ti et al., 2003; Chazan et al., 2004). In the present study, E. coli was the main uropathogen in $38 \%$ of samples and it was also more prevalent in females (49\%) (Table 1). The anatomical differences in the female urinary system, including a short urethra and the proximity to the vulvar and perianal areas, result in a higher prevalence of UTI in females (Sobel and
Kaye 2010). The guidelines of the Infectious Diseases Society of America (IDSA) for the treatment of UTI recommend avoiding empirical treatment with a specific antibiotic when the local level of resistance among $E$. coli strains exceeds 20\% (Warren et al., 1999). In previous, the first empirical antibiotic for UTI treatment was SXT. However, resistance rate to SXT in E. coli was reached at $50 \%$ in several Korean studies (Ko et al., 2003; Shin et al., 2005) and was $36.06 \%$ in this study. And these finding demonstrated that SXT was no longer reasonable choice in empirical treatment of UTI. In addition, the most alarming finding in our study was the exceedingly high resistance rate of $E$. coli to ciprofloxacin (33.55\% in 2012 3) (Fig. 1). This rate is lower than that reported in Shin et al. (2005) but higher than that of Cullen et al. (2013). These results imply that quinolones, which are commonly used in the management of UTI, might gradually lose their utility in the empiric treatment of UTI. In addition, the resistance rates for firstgeneration and third-generation cephalosporins in E. coli increased over time (Fig. 1). ESBL-producing $E$. coli is an emerging cause of nosocomial healthcare-associated, and community-acquired infection worldwide (Jacobby and Medeiros, 1991; Bush, 2001). Inadequate empirical antibiotic therapy for infections caused by this microorganism is associated with poor outcomes and the use of carbapenem or cefepime is only effective for patients infected by ESBL (Ramphal and Ambrose, 2006). In addition, although imipenem-resistant isolates were not observed in this study, isolates producing carbapenemase, such as NDM, and KPC (Nordmann et al., 2011; Kim et al., 2013), have recently been reported worldwide. Therefore more constricted surveillance is required in the clinical laboratory.

The incidence of vancomycin-intermediate $S$. aureus (VISA) and VRSAs is in an increasing trend globally, including in Korea. In a nationwide surveillance study of VISA in Korean hospital from 2001 to 2006 (Chung et al., 2010), the prevalence of VISA was $0.09 \%(33 / 37,856$ MRSA). In Asia, VISA prevalence is in Thailand (0.3 $2.3 \%$ ) and Japan ( $0.24 \%)$. In this study, MRSA is 570 isolates (73\% of 784 S. aureus) for 10 yrs. Therefore, estimated prevalence of VISA may be 0.5 isolate $(0.09 \%$ of 570 isolates) and both VRSA and VISA were not observed in 
this study. However, because the E-test or broth dilution methods are required to confirm VISA, the routine antibiotic susceptibility testing (disk diffusion or Vitek) had a possibility of non-detecting VISA.

For the treatment of enterococcal infection, ampicillin and aminoglycosides are the drugs of choice. Diseases caused by strains resistant to ampicillin and aminoglycosides can be treated with vancomycin. In the present study, vancomycin resistance in E. faecium was $20.03 \%$ over a period of 10 years and vancomycin resistance in E. faecium increased from $15.69 \%$ in $2004 \sim 5$ to $25.00 \%$ in $2012 \sim 3$. The occurrence of VRE represents a serious problem because the treatment of infections provoked by VRE may be highly problematic because the choice of suitable medicaments is mainly limited to linezolid and quinupristin/dalfopristin, or due to the transmission of vancomycin resistant genes, such as $v a n A$ or $v a n B$. A significant correlation between vancomycin use and VRE occurrence has been demonstrated (Quale et al., 1996; Empey and Rapp, 2002; Kolar et al., 2002) and the use of infection control measures including frequent hand washing, has been successful for containing small monoclonal outbreaks (Boyce et al., 1994). Therefore, the VRE problem requires not only improved hygiene in clinical department but also the responsible use of antibiotics.

The $P$. aeruginosa was highly resistant to most antibiotics and recently, an increase in imipenem-resistant $P$. aeruginosa (IRPA) has become a serious problem. In the present study, the prevalence IRPA was $33.33 \%$ during 2004 5 and after that time, was decreased to $21 \sim 24 \%$. IRPA usually causes various nosocomial infections in long term hospitalized patients. However, Shin et al. (2005) reported that IRPA in even CA-UTI was $47 \%$ in $2001 \sim 3$. Moreover, metallo- $\beta$ lactamase (MBL; class B $\beta$-lactamase defined by Ambler), the most important resistance mechanism to imipenem in $P$. aeruginosa, was easily transmitted to other isolates using various genetic element, such as integrin (Poirel et al., 2000; Lee et al., 2002, Shin et al., 2008). Therefore, rapid detection or strict control of the transmission of the MBL producer is also required.

This study had several limitations due to the fact that it was a retrospective study. First, the results of the antimicrobial susceptibility testing obtained from conventional disk diffusion or the Vitek II system were confirmed by the E-test only in isolates exhibiting resistance to vancomycin or imipenem. Second, we did not interpret the positive cultures in terms of contamination or clinically relevant infection. Third, we included bacteria isolated from both CA-UTIs and hospital associated UTI due to difficulties associated with the classification of clinical infection, which demonstrated different distributions of pathogens and antimicrobial susceptibility patterns.

In conclusion, over the last 10 years, the proportions of ciprofloxacin resistant E. coli and multidrug-resistant bacteria, including ESBL and VRE, have significantly increased. These findings demonstrate the need for strict control over the use of antibiotics and indicate the need for updated guidelines for the treatment of UTIs.

\section{Acknowledgements}

This study was supported by the research grant of Chungbuk National University in 2013.

\section{Conflict of interest}

The authors have no conflicts of interest to disclose.

\section{REFERENCES}

Boyce JM, Opal SM, Chow JW, Zervos MJ, Potter-Bynoe G, Sherman CB. Outbreak of multidrug-resistant Enterococcus faecium with transferable $v a n B$ class vancomycin resistance. J Clin Microbiol. 1994. 32: 1148-1153.

Bush K. New $\beta$-lactamases in gram-negative bacteria: diversity and impact on the selection of antimicrobial therapy. Clin Infec Dis. 2001. 32: 1085-1089.

Chazan B, Sakran W, Raz R, Colodner R. Improved antimicrobial susceptibility of community-acquired uropathogens in northern Israel (1995-1999-2002). Int J Antimicrob Agents. 2004. 24: 89-92.

Chung G, Cha J, Han S, Jang H, Lee K, Yoo J, Yoo J, Kim H, Eun S, Kim B, Park O, Lee Y. Nationwide surveillance study of vancomycin-intermediate Staphylococcus aureus strains in Korean hospitals form 2001 to 2006. J Microbiol Biotechnol. 2010. 20: 637-642.

Clinical and Laboratory Standards Institute. Performance standards for antimicrobial susceptibility testing: twenty two infor- 
mational supplement (M100-S22). Wayne, PA: CLSI, 2012.

Cullen IM, Manecksha RP, McCullagh E, Ahmad S, O'Kelly F, Flynn R, McDermott TED, Murphy P, Grainger R, Fennell JP, Thornhill JA. An 11-year analysis of the prevalent uropathogens and the changing pattern of Escherichia coli antibiotic resistance in 38,530 community urinary tract infections, Dublin 1999-2009. Ir J Med Sci. 2013. 182: 81-89.

den Heijer CD, Donker GA, Males J, Stobberingh EE. Antibiotic susceptibility of unselected uropathogenic Escherichia coli from female Dutch general practice patients: a comparison of two surveys with a 5 year interval. J Antimicrob Chemother. 2010. 65: 2128-2133.

Empey KM, Rapp RP, Evans ME. The effect of an antimicrobial formulary change on hospital resistance patterns. Pharmacotherapy. 2002. 22: 81-87.

Farrell DJ, Moorssey I, De Rubeis D, Robbins M, Felmingham D. A UK multicentre study of the antimicrobial susceptibility of bacterial pathogens causing urinary tract infection. J Infect 2003. 46: 94-100.

Freidrich LV, White RL, Bosso JA. Impact of use of multiple antimicrobials on changes in susceptibility of gram-negative aerobes. Clin Infect Dis. 1999. 28: 1017-1024.

Gupta K, Hooten TM, Woobe CL, Stamm WE. The prevalence of antimicrobial resistance among uropathogens causing acute uncomplicated cystitis in young women. Int $\mathbf{J}$ Antimicrob Agents. 1999. 11: 305-308.

Gupta K Addressing antibiotic resistance. Am J Med. 2002. 113(s): 29S-34S.

Hooton TM, Stamm WF. Diagnosis and treatment of uncomplicated urinary tract infection. Infect Dis Clin N Am 1999. 11: 551 -581 .

Jacoby GA, Medeiros AA. More extended-spectrum $\beta$-lactamase. Antimicrob Agents Chemother. 1991. 35: 1697-1704.

Jones RN, Kugler KC, Pfaller MA, Winokur PL. Characteristics of pathogens causing urinary tract infections in hospital in North America: results from the SENTRY antimicrobial surveillance program, 1997. Diagn Microbiol Infect Dis. 1999. 35: 55-63.

Kahlmeter G. An international survey of the antimicrobial susceptibility of pathogens from uncomplicated urinary tract infection: the ECO.SENS Project. J Antimicrob Chemother. 2003. 51: 69-76.

Karlowsky JA, Kelly LJ, Thornsberry C, Jones ME, Sahm DF. Trends in antimicrobial resistance among urinary tract infection isolates of Escherichia coli from female outpatients in the United States. Antimicrob Agent Chemother. 2002. 48: 2540
-2545 .

Kim SY, Shin J, Shin SY, Ko KS. Characteristics of carbapenemresistant Enterobacteriaceae isolates from Korea. Diagn Microbiol Infect Dis 2013. 76: 486-490.

Ko YH, Oh JS, Cho DY, Bea JH, Koh SK. Change of causative organisms and antimicrobial sensitivity of urinary tract infection between 1979 and 2001. Korean J Urol. 2003. 44: 342 -350 .

Kolar M, Vagnerova I, Latal T, Urbanek K, Typovska H, Hubacek J, Papajik T, Raida L, Faber E. The occurrence of vancomycinresistant enterococci in hematological patients in relation to antibiotic use. New Microbiol. 2002. 25: 373-379.

Lee K, Lim JB, Yum JH, Yong D, Chong Y, Kim JM, Livermore DM. bla $a_{\mathrm{VIM}-2}$ cassette-containing novel integrons in metallo$\beta$-lactamase-producing Pseudomonas aeruginosa and Pseudomonas putida isolates disseminated in a Korean hospital. Antimicrob Agent Chemother. 2002. 46: 1053-1058.

Nordmann P, Nass T, Poirel L. Global spread of carbapenemaseproducing Enterobactericeae. Emerg Infect Dis. 2011. 17: 1791-1798.

Poirel L, Naas T, Nicolas D, Collet L, Bellais S, Cavallo JD, Nordmann P. Characterization of VIM-2, a carbapenemhydrolyzing metallo- $\beta$-lactamase and its plasmid- and integrinbone gene from a Pseudomonas aeruginosa clinical isolate in France. Antimicrob Agent Chemother. 2000. 44: 891-897.

Quale J, Landman D, Atwood E, Kreiswirth B, Willey BM, Ditore V, Zaman M, Patel K, Saurina G, Huang W, Oydna E, Burney S. Experience with a hospital-wide outbreak of vancomycin resistant enterococci. Am J Infect Control. 1996. 24: 373-379.

Ramphal R, Ambrose PG. Extended-spectrum $\beta$-lactamases and clinical outcomes: current data. Clin Infect Dis 2006. 42: S164 -S172.

Ronald A. The etiology of urinary tract infection: traditional and emerging pathogens. Dis Mon. 2003. 49: 71-82.

Shin KS, Son BR, Hong SB, Kim J. Dipicolinic acid-based disk methods for detection of metallo- $\beta$-lactamase-producing Pseudomonas spp. and Acinetobacter spp. Diagn Microbiol Infect Dis. 2008. 62: 102-105.

Shin JW, Kim HR, Lee HR, Chung J, Min K, Moon CS, Ryu SM, Lee JN. Etiology and antimicrobial susceptibility of bacterial pathogen causing community-acquired urinary tract infection at a tertiary-care hospital. Korean J Clin Microbiol 2005. 8: 142-147.

Sobel JD, Kaye D. Urinary tract infections: principles and practice of infectious disease (Mandell GL, Bennett JE, Dolin R eds). 
2010. $7^{\text {th }}$ ed. pp. 957-985. Churchill Livingstone Elsevier Press. Philadelphia, PA, USA.

Thomson KS, Sanders WF, Sanders CC. USA resistance patterns among UTI pathogens. J Antimicrob Chemother. 1994. 33(s): 9S-15S.

Ti TY, Kumarasinghe G, Taylor MB, Tan SL, Ee A, Chua C, Low A. What is true community-acquired urinary tract infection? Comparison of pathogen identified in urine from routine outpatient specimens and from community clinics in a prospective study. Eur J Clin Microbiol Infect Dis. 2003. 22: 242 -245 .
Turnidge J, Bell J, Biedenbach DJ, Jones RN. Pathogen occurrence and antimicrobial resistance trend among urinary tract infection isolates in the Asia-Western Pacific region: report from the SENTRY antimicrobial surveillance program, 1998 1999. Int J Antimicrob Agents. 2002. 20: 10-17.

Warren JW. Practice guidelines for the treatment of uncomplicated cystitis. Curr Urol Rep. 2001. 2: 326-329.

Warren JW, Abrutyn E, Habel JR, Johnson JR, Schaeffer AJ, Stamm WE. Guidelines for antimicrobial treatment of uncomplicated acute bacterial cystitis and acute pyelonephritis in women. Clin Infect Dis. 1999. 29: 745-758. 